Rev. Elev. Méd. vét. Pays trop., 1973, 26 (4) : 13 a - 19 a

\title{
Les helminthes parasites des thons tropicaux
}

\author{
par J. BUSSIERAS $\left({ }^{*}\right)$ et F. BAUDIN-LAURENCIN (**)
}

\begin{abstract}
RESUME
Les auteurs présentent une brève récapitulation des helminthes récoltés sur les thons de l'Atlantique tropical; ils essaient ensuite de montrer l'intérêt, tant pratique que théorique, de telles études.
\end{abstract}

Il n'est pas possible à l'époque actuelle d'établir une récapitulation complète des espèces d'helminthes parasites des thons tropicaux, et cela pour deux raisons :

- la systématique exacte des thons est encore fort mal précisée, le nombre des espèces décrites étant certainement trop élevé. Il semble en particulier que certains thons, à répartition sans doute cosmopolite, ont reçu des noms spécifiques différents, en fonction surtout de leur origine géographique, ou de très faibles variations morphologiques;

- chez ces hôtes souvent mal identifiés, ont été décrites de nombreuses espèces d'helminthes, parfois tellement voisines que l'on retenait justement la différence d'hôte comme critère d'identification.

Une comparaison à l'échelle mondiale, aussi bien des poissons que de leurs parasites, sera donc nécessaire.

C'est pourquoi nous nous limiterons aux parasites récoltés sur les thons de l'Atlantique centre-est, et en considérant essentiellement ceux des poissons les plus fréquemment pêchés dans cette zone et ramenés à Abidjan ou à Dakar.

Le terme même de thon est très imprécis, car il peut servir à désigner :

— soit l'ensemble de la famille des Thunnidae (d'où l'on retire parfois une famille des Katsuwonidae); genres);

- soit le seul genre Thunnus (lui-même parfois démembré en plusieurs genres ou souss-

- soit les seules espèces ayant légalement droit à l'appellation " thon " en conserverie, et dont la liste est sujette à modifications.

Pratiquement, les principaux "thons" que l'on peut étudier dans l'Atlantique centre-est appartiennent aux espèces :

Thunnus albacares (Bonnaterre, 1788), l'albacore;

Thunnus obesus (Lowe, 1839), le patudo;

Katsuwonus pelamys (L.), le listao;

(*) Professeur à l'Ecole Inter-Etats des Sciences et Médecine Vétérinaires, Dakar, Sénégal.

(**) Centre de Recherches Océanographiques, Abidjan, Côte d'Ivoire. 
Euthynnus alleteratus (Rafinesque, 1810), la thonine, plus rarement examinée parce que non utilisée en conserverie.

En Afrique occidentale, seuls les albacores ont fait l'objet de recherches parasitaires complètes et systématiques (2). Les autres thons n'ont été examinés qu'incidemment ou partiellement.

\section{PRINCIPALES ESPECES PARASITES}

\subsection{Cestodes}

Tous les Cestodes observés et qui ont pu être déterminés appartiennent au groupe des Tétrarhynques (= ordre des Trypanorhyncha).

\subsubsection{Tentaculariidae}

- Tentacularia coryphenae Bosc, 1802.

Hôtes: le listao, Katsuwonus pelamys, parfois l'albacore, Thunnus albacares.

Stade : postlarve.

Localisation: péritoine et paroi musculaire de l'abdomen; un exemplaire retrouvé chez le listao à la surface des branchies.

\subsubsection{Hepatoxylidae}

- Hepatoxylon trichiuri Holten, 1802.

Hôtes : l'albacore, $T$. albacares, et le listao, K. pelamys.

Stade : postlarve.

Localisation: dans la cavité générale, ou sous le péritoine viscéral (paroi gastrique).

\subsubsection{Sphyriocephalidae}

- Sphyriocephalus dollfusi Bussiéras et Aldrin, 1968.

Hôte : le patudo, $T$. obesus.

Stade : postlarve.

Localisation: seuls deux spécimens sont connus, récoltés à Abidjan dans la cavité gastrique d'un patudo.

\subsubsection{Dasyrhynchidae}

- Dasyrhynchus talismani Dollfus, 1935.

. Hôtes : l'albacore T. albacares, et le patudo, T. obesus.

Stade: larve plerocercus.

Localisation : appareil circulatoire, dans les artères issues du tronc coeliaco-mésentérique d'une part (7) et dans les artères branchiales afférentes d'autre part (2). Cette double localisation n'est d'ailleurs pas sans poser certains problèmes d'interprétation.

- Callitetrarhynchus gracilis (Rudolphi, 1819).

Hôtes : la thonine, E. alleteratus, et l'albacore, $T$. albacares.

Stade : larve plerocercus.

Localisation : kystes péritonéaux. 
1.1.5. La présence de larves de Cestodes non identifiés est fréquemment constatée dans l'aorte dorsale. Noyés dans le tissu conjonctif réactionnel, ces éléments rubanés peuvent atteindre plusieurs dizaines de centimètres de long.

\subsection{Monogènes}

\subsubsection{Capsalidae}

- Nasicola klawei (Stunkard, 1962).

Hôte : l'albacore, T. albacares.

Localisation: sacs nasaux, dans chacun desquels on retrouve le plus souvent deux vers.

Discussion : Caballerocotyla klawei se différenciant nettement des autres espèces du genre, c'est à juste titre, semble-t-il, que YAMAGUTI (14) a créé pour elle le nouveau genre Nasicola.

Par ailleurs, dans l'Atlantique oriental, N. klawei a, depuis les observations de ROSSIGNOL et REPELIN (12), toujours été considéré comme spécifique de l'albacore : le parasite est retrouvé chez pratiquement 100 p. 100 des albacores examinés, et jamais chez les autres thons (8). C'est pourquoi il est très curieux qu'il ait récemment été signalé :

dans le Pacifique, chez Thunnus sibi (espèce très voisine de $T$. obesus, peut-être même identique) (14);

dans l'Atlantique occidental, chez Thunnus atlanticus (1).

- Caballerocotyla abidjani Bussiéras et Baudin-Laurencin, 1970.

Hôte : l'albacore, $T$, albacares.

Localisation: face interne des opercules.

Discussion : cette espèce est généralement observée sous la forme C.a. abidjani; beaucoup plus rarement on rencontre la sous-espèce C.a. microcotyle, à l'opisthapteur très petit. Ces deux sous-espèces apparaissent respectivement très proches de Capsala neothunni Yamaguti, 1968, et de Capsala biparasitica (Goto, 1894), selon la description de YAMAGUTI (1968) (qui, pour C. biparasitica, diffère quelque peu de celle de GOTO), s'en distinguant surtout par les dimensions et par le nombre de pointes des épines de la face dorsale; une comparaison minutieuse des spécimens semble nécessaire.

- Caballerocotyla pseudomagronum Bussiéras, 1972.

Hôte : le patudo, T. obesus.

Localisation: cavité buccale (langue et palais).

Discussion : cette espèce est sans doute identique à Capsala gotoi YAMAGUTI, 1968, ellemême récoltée sur les branchies de $T$. sibi, le thon patudo du Pacifique; il serait bon de comparer les divers spécimens entre eux, et avec l'espèce très voisine Caballerocotyla magronum (Ishii, 1936).

- Caballerocotyla verrucosa Bussiéras, 1972.

Hôte : $T$. obesus et $T$. albacares.

Localisation : cavité buccale (langue et palais), ainsi que, chez $T$. obesus, face interne des opercules et branchies.

Discussion : cette espèce sera à comparer avec des spécimens beaucoup plus petits, récoltés chez Thunnus thynnus et Euthynnus affinis de la mer de Chine, de l'espèce Caballerocotyla paucispinosa Mamaev, 1968. En outre, elle présente des analogies avec Caballerocotyla nozawae (Goto, 1894), du moins selon la description de YAMAGUTI (1968) (qui indique que l'opisthapteur est couvert de papilles, et que les testicules sont généralement limités au champ inter- 
intestinal, alors que dans la description initiale de GOTO, les papilles de l'opisthapteur ne sont pas mentionnées, et les testicules sont dits s'étendant quelque peu dans les champs latéraux).

\subsubsection{Hexostomatidae}

\section{- Hexostoma sp.}

Hôtes: $T$. obesus et $T$. albacares.

Localisation : branchies.

Discussion: depuis la description de $H$. thynni (de la Roche, 1811) des branchies de $T$. thynnus, $T$. obesus et Sarda sarda, de nombreuses autres espèces ont été signalées chez divers thons, souvent caractérisées par de faibles différences morphologiques qui, d'après les plus récentes études, paraissent être le fait de variations individuelles. Il semble que les spécimens récoltés dans le Golfe de Guinée peuvent être rattachés à l'espèce $H$. thynni, de même d'ailleurs que $H$. sibi, récemment décrit par YAMAGUTI (14) chez le patudo et l'albacore du Pacifique.

\section{- Neohexostoma sp.}

Hôte: T. albacares.

Localisation : branchies.

Discussion : compte tenu des variations individuelles également constatées dans sa morphologie, ce parasite semble identique à $N$. kawakawa décrit par YAMAGUTI (1968) chez $T$. macropterus, et à $N$. euthynni (Meserve, 1938).

\subsubsection{Gastrocotylidae}

\section{- Allopseudaxine sp.}

Hôte : $T$. albacares.

Localisation : branchies.

Discussion : cette espèce semble très proche de $A$. yaito et $A$. macrova, parasites des thonines (Euthynnus sp.) indo-pacifiques.

- Sibitrema poonui Yamaguti, 1968.

Hôte : T. albacares.

Localisation: branchies.

Discussion : ce parasite a été récolté à Abidjan, sur des thons pêchés à proximité de l'Ile de la Tortue (mer des Antilles). Sur 6 albacores examinés, 5 présentaient ce monogène. Il est intéressant de noter que ce ver, jamais mis en évidence dans l'Atlantique Centre-Est, a été signalé par YAMAGUTI chez l'albacore et le patudo d'Hawaii.

\subsection{Trématodes digènes}

\subsubsection{Sanguinicolidae}

- Cardicola sp.

Hôte: $T$. albacares.

Localisation: trouvé dans les eaux de rinçage des branchies; probablement d'origine sanguine.

Discussion : un seul individu a pu être mis en évidence dans le Golfe de Guinée. Il semble identique à Cardicola ahi YAMAGUTI, 1968, rencontré aux Hawaii à la fois chez T. macropterus et $T$. sibi. 


\subsubsection{Hirudinellidae}

- Uroproctinella spinulosa (Yamaguti, 1936).

Hôte : $T$. albacares.

Localisation : estomac.

Discussion : ce parasite, trouvé par YAMAGUTI chez $T$. alalunga, est rencontré en Afrique tropicale chez $T$. albacares, principalement au large d'Abidjan.

\subsubsection{Gorgoderidae}

- Phyllodistomum thunni Baudin-Laurencin et Richard, 1973.

Hôte : $T$. albacares.

Localisation : reins, uretères, vessie.

Discussion : relativement fréquent chez les jeunes thons, le ver n'a jamais été rencontré sur des albacores de plus de $110 \mathrm{~cm}$ (environ 3 ans).

\subsubsection{Didymozoidae}

Dans toutes les espèces de thons examinés, ont été récoltés de nombreux spécimens de Didymozoidae, appartenant en particulier aux genres Didymozoon, Koellikeria, Didymocystis, Nematobothrium, Lobatozoum; une étude détaillée sera nécessaire pour préciser les espèces en cause.

\subsection{Nématodes}

\subsubsection{Anisakidae}

Des formes immatures sont parfois récoltées dans le péritoine de $T$. albacares, T. obesus, $K$. pelamys, E. alleteratus.

\subsubsection{Camallanidae}

- Oncophora albacarensis Baudin-Laurencin, 1972.

Hôte : $T$. albacares.

Localisation : organe pylorique.

Discussion: la seule autre espèce connue du genre, Oncophora melanocephala (Rudolphi, 1819), avait été très anciennement mise en évidence (intestin, organe pylorique, vésicule biliaire) chez divers Scombridae méditerranéens et chez un thon rouge ( $T$. thynnus) pêché au Brésil.

\subsubsection{Philometridae}

D'espèce indéterminée, ils sont retrouvés dans les ovaires, notamment chez $T$. albacares.

\subsection{Acanthocéphales}

- Rhadinorhynchus cadenati Golvan et Houin, 1964.

- Bolbosoma vasculosum (Rudolphi, 1819).

Hôtes : $T$. albacares, $E$. alleteratus, et nombreuses espèces appartenant à diverses familles de poissons marins.

Localisation : appareil digestif.

Discussion: récoltés principalement dans la région nord de l'Atlantique tropical oriental. 


\section{INTERET DE L'ETUDE HELMINTHOLOGIQUE DES THONS}

\subsection{Correction de vieilles erreurs d'identification}

En 1819, RUDOLPHI classait dans la famille des Camallanidae le mâle d'Oncophora melanocephala, mais considérait la femelle (décrite à partir de matériel incomplet) comme un trichure, sous l'appellation de Trichocephalus gibbosus. L'étude de $O$. albacarensis a permis de remédier à cette erreur.

\subsection{Compréhension du cycle de certains helminthes}

Dasyrhynchus talismani n'était connu que sous sa forme adulte, trouvée dans l'intestin du requin bleu, Prionace glauca; aussi, la connaissance de la présence des larves plerocercus chez $T$. albacares et $T$. obesus permet-elle de comprendre une partie du cycle de ce tétrarhynque, les thons étant dévorés par les requins. Il reste bien entendu à découvrir les invertébrés premiers hôtes intermédiaires.

Inversement, il arrive que l'étude des helminthes des thons conduise à identifier d'autres stades des mêmes parasites, chez divers poissons. C'est ainsi qu'ont été retrouvées des formes adultes de Tentacularia coryphaenae et de Dasyrhynchus talismani dans l'intestin du requin Carcharhinus falciformis; il s'agit là, semble-t-il, d'un hôte nouveau pour ces espèces.

\subsection{Diagnose rapide des espèces de poissons}

Dans l'Atlantique tropical oriental, Nasicola klawei a été retrouvé chez pratiquement tous les albacores examinés et n'est jamais rencontré dans d'autres espèces.

Or, il est parfois difficile de distinguer les formes jeunes d'albacores $T$. albacares, de celles des patudos, $T$. obesus. C'est pourquoi la recherche de la présence ou de l'absence de ce Monogène avait été considérée comme un moyen sûr d'identification de ces deux thons, adopté dans des ouvrages récents sur la systématique des poissons tropicaux (5).

Cette notion devra cependant être confirmée, depuis que YAMAGUTI (14) a retrouvé ce même Monogène chez Thunnus sibi, le patudo du Pacifique. Mais peut-être faudra-t-il vérifier l'identification même de cette dernière espèce-hôte, dont les formes jeunes risquent certainement de prêter à confusion avec celles de $T$. macropterus.

\subsection{Relations entre les espèces de thons des différents océans}

Les thons d'un même type ont généralement reçu des noms différents en fonction de la zone géographique considérée. Dans le cas du thon albacore, ont ainsi été décrites jusqu'à six espèces différentes (10).

Le plus souvent cependant, pour l'albacore comme pour le patudo, on tend à reconnaittre deux espèces, l'une atlantique, l'autre indo-pacifique.

\begin{tabular}{|l|l|l|}
\hline & \multicolumn{1}{|c|}{ Espèces atlantiques } & \multicolumn{1}{c|}{ Espèces indo-pacifiques } \\
\hline Albacore & T. albacares (Bonnaterre, 1788) & $\begin{array}{l}T \text {. macropterus } \text { (Temminck et Schlegel, } \\
1842 \text { ) }\end{array}$ \\
Patudo & T. obesus (Lowe, 1839) & sibi (Schlegel, 1844) \\
\hline
\end{tabular}

En réalité, en Afrique australe a été observée (13) pendant l'été une continuité de peuplement en albacores et patudos entre les versants atlantique et indien, la séparation en deux populations distinctes ne se faisant que pendant l'hiver.

Ce fait conduit à suspecter l'existence d'une seule espèce d'albacore, et d'une de patudo, à distribution mondiale.

L'étude, et surtout la comparaison, des helminthes parasites récoltés sur des thons de diverses origines, apportera des arguments intéressants dans cette mise au point de la systématique de ces poissons. 


\subsection{Identification de populations de poissons}

Pour les seuls albacores du Golfe de Guinée, on constate que des parasites sont plus nombreux dans certaines zones de pêche. Ainsi, les Acanthocéphales apparaissent plus fréquemment dans la région du Nord (Dakar), alors que les Uroproctinella y sont plus rarement rencontrés qu'ailleurs. Si l'on considère que ces parasites demeurent suffisamment longtemps chez leurs hôtes, il faut en conclure que les albacores pêchés au large du Sénégal ne migrent pas dans le reste du Golfe.

Une étude faisant appel à des méthodes d'analyse factorielle, et portant sur l'ensemble de la faune parasitaire, confirme l'existence chez l'albacore de l'Atlantique oriental de deux grands groupes, deux populations géographiquement distinctes.

Les résultats permettent des études plus précises de la dynamique des stocks, et, par suite, un contrôle plus efficace de la pêche de l'albacore, poisson qui, du fait de son importance économique, pourrait être et est peut-être déjà surexploité.

\section{SUMMARY}

\section{The Helminths parasites of tropical Tunas}

The authors record a list of the helminths collected in the tropical Atlantic tunas. They then emphasize the interest, as practical as theoretic, of such studies.

\section{RESUMEN}

Los helmintos parasitos de atunes tropicales

Los autores dan una recapitulación sucinta de los helmíntos recogidos en los atunes de Atlántico tropical; luego trátan del interés, tanto práctico como teorico, de tales estudios.

\section{BIBLIOGRAPHIE}

1. BANE (G.W.). Parasites of the Yellowfin Tuna, Thunnus albacares, in the Atlantic Ocean (Pisces: Scombridae), Wasmann. J. Biol., 1969, 27 (1) : 163-175.

2. BAUDIN-LAURENCIN (F.). Crustacés et Helminthes parasites de l'Albacore (Thunnus albacares) du Golfe de Guinée - Note préliminaire. Doc. Scient. Centre Rech. Océanogr. Abidjan, 1971, 2 (1-2): 11-30.

3. BAUDIN-LAURENCIN (F.). Oncophora albacarensis n. sp., Nématode Camallanidae, parasite du Thon Albacore) Thunnus albacares. Bull. Muséum nat. Hist. nat., 1972, 3e sér. (96). Zoologie (75): 1329-1332.

4. BAUDIN-LAURENCIN (F.) et RICHARD (J.). Phyllodistomum thunni n. sp. (Trematoda, Gorgoderidae), trématode parasite du thon albacore Thunnus albacares. Bull. Muséum nat. Hist. nat., 1973 (sous presse).

5. BLACHE (J.), CADENAT (J.) et STAUCH (A.). Clés de détermination des poissons signalés dans l'Atlantique tropical oriental. Paris, O.R.S.T.O.M., 1970, 480 p.

6. BUSSIERAS (J.). Les Monogènes Capsalinae parasites des thons de l'Atlantique tropical oriental. Ann. Parasit. hum. comp., 1972, 47 (1) : 29-49.

7. BUSSIERAS (J.) et ALDRIN (J.F.). Une tétrarhynchose vasculaire des thons du Golfe de Guinée, due aux larves plerocercus de Dasyrhynchus talismani R. Ph. Dollfus, 1935. Rev. Elev. Méd. vét. Pays trop., 1965,18 (2): $137-143$.

8. BUSSIERAS (J.) et ALDRIN (J. F.). Caballerocotyla klawei Stunkard, 1962, Monogène parasite des sacs nasaux du thon albacore de l'Océan Atlantique. Rev. Elev. Méd. vét. Pays trop., 1967, 20 (1) : $105-108$.

9. BUSSIERAS (J.) et BAUDIN-LAURENCIN (F.). Caballerocotyla abidjani n. sp. (Monogenea, Capsalidae, parasite des opercules du thon albacore, Thunnus albacares. Cah. O.R.S.T.O.M., sér. Océanogr, 1970, 8 (3): 47-51.

10. JORDAN (D.S.) et EVERMANN (B.W.). A review of the giant mackerel-like fishes, tunnies, spearfishes and swordfishes. Occ. Pap. Calif. Acad. Sci., 1926 (12): 1-113.

11. MAMAEV (Y,L.). Helminthes des thons du sud de la mer de Chine (en russe). In: SKRJABIN et MAMAEV : Helminthes des animaux de l'Océan Pacifique. Moscou, Izdat. Nauka, 1968, pp. 5-27.

12. ROSSIGNOL (M.) et REPELIN (R.). Présence d'un trématode parasite des sacs nasaux chez $N$. albacora (Lowe). Travaux Centre Océanogr. Pointe Noire, 1962, 2:177 et 288.

13. TALBOT (F.S.) et PENRITH (M. J.). Tunnies and marlins of South Africa. Nature, London, 1962,193 (4815) : 558-559.

14. YAMAGUTI (S.). Monogenetic Trematodes of Hawaiian Fishes. Univ. of Hawaii Press, 1968,288 p.

15. YAMAGUTI (S.). Digenetic Trematodes of Hawaiian Fishes. Tokyo, Keigaku Publ., 1970, 264 p. 